Relations industrielles

Industrial Relations

\title{
La rémunération des enseignants
}

\section{Bertrand Belzile}

Volume 34, numéro 2, 1979

URI : https://id.erudit.org/iderudit/028966ar

DOI : https://doi.org/10.7202/028966ar

Aller au sommaire du numéro

\section{Éditeur(s)}

Département des relations industrielles de l'Université Laval

\section{ISSN}

0034-379X (imprimé)

1703-8138 (numérique)

Découvrir la revue

Citer cet article

Belzile, B. (1979). La rémunération des enseignants. Relations industrielles /

Industrial Relations, 34(2), 335-345. https://doi.org/10.7202/028966ar

Tous droits réservés @ Département des relations industrielles de l'Université Laval, 1979
Ce document est protégé par la loi sur le droit d'auteur. L’utilisation des services d'Érudit (y compris la reproduction) est assujettie à sa politique d'utilisation que vous pouvez consulter en ligne.

https://apropos.erudit.org/fr/usagers/politique-dutilisation/ 


\section{COMMENTAIRES}

\section{La rémunération des enseignants}

\section{Bertrand Belzile}

Monsieur Michael Bell, du Département des activités sectorielles du Bureau international du travail (B.I.T.), a complété une étude très documentée sur la rémunération des enseignants dans plusieurs pays. ${ }^{1}$ Le contenu le plus intéressant se retrouve dans la deuxième partie où l'auteur traite de la structure des traitements et des suppléments de traitement et où il effectue aussi un essai de comparaison internationale relativement aux gains des enseignants au cours des dernières années. Un peu en guise d'introduction à l'objet principal de l'étude, l'auteur consacre la première des deux parties du volume à expliquer les méthodes selon lesquelles la rémunération des enseignants est déterminée. Dans le présent texte, nous allons faire état plutôt rapidement de cette première partie, pour nous attarder davantage à la deuxième.

Une remarque capitale s'impose à ce moment-ci: l'ouvrage du B.I.T. ne recense pas de façon exhaustive les politiques de rémunération prises individuellement, mais donne plutôt ce qui semble être une bonne idée de chaque composante de telles politiques. Autrement dit, l'ouvrage en question n'est pas articulé d'abord autour des pays, mais autour des politiques et même des composantes de celles-ci. En conséquence, il apparaît impossible de comparer les pays entre eux au niveau de la rémunération totale; la comparaison peut se faire uniquement au niveau de certaines composantes de la rémunération. Afin de pallier cette lacune, nous essayerons de situer le Québec par rapport aux autres pays, en complétant l'information pertinente pour le Québec pour chaque composante, dans la mesure du possible. Les résultats d'un tel exercice doivent être utilisés avec beaucoup de prudence, étant donné que ce qui compte en dernier ressort, c'est le niveau relatif de la rémunération totale, pour ne pas dire globale, et non le niveau relatif de chaque composante de la rémunération. Autrement dit, l'avantage d'un pays sur une composante peut être annulé par un désavantage sur une autre composante.

* BELZILE, Bertrand, directeur, service des études économiques, ministère de l'Education.

** Nous tenons à remercier M. Michel Bergeron, du Service des Relations de travail du Ministère, de nous avoir suggéré certaines précisions relativement aux conditions de travail des enseignants du Québec.

1 Bureau international du travail, Genève, 1978, $128 \mathrm{pp}$. 


\section{CHAPITRE 1: SELON QUELLES MÉTHODES LA RÉMUNÉRATION DES ENSEIGNANTS EST-ELLE DÉTERMINÉE?}

Cette question générale en appelle deux particulières. La première a trait aux mécanismes et procédures qui président à la détermination du niveau des traitements et l'autre aux critères en usage. L'intervention des enseignants, dans le processus de détermination de leurs traitements, constitue l'élément-clé dans les mécanismes et procédures en question. A cet égard, qu'il suffise de rappeler la conclusion générale de l'auteur: "Premièrement, il apparaît que les pays où les enseignants ne sont pas consultés au sujet du niveau de leurs traitements sont en minorité. Deuxièmement, si dans la majorité des pays et systèmes scolaires où les organisations d'enseignants ont leur mot à dire, leur rôle est purement consultatif (la décision finale demeurant l'apanage des autorités gouvernementales), il en est un nombre appréciable où les traitements des enseignants sont déterminés par un système de négociation collective ou par des arbitrages dont l'issue lie les deux parties". (p. 17) Il va sans dire que le Québec se situe parmi ce dernier groupe de pays.

\section{CHAPITRE 2: QUELS CRITÈRES SONT EN USAGE DANS LA DÉTERMINATION DU NIVEAU DE RÉMUNÉRATION DES ENSEIGNANTS?}

Ce chapitre n'apprendra que très peu de choses au lecteur le moindrement au courant des questions salariales chez les enseignants. Le sujet est pourtant bien couvert, aussi bien quant aux critères retenus qu'à la présentation qui est faite de chacun d'eux. Les critères sont classifiés de façon usuelle en internes et externes, les seconds étant plus décisifs que les premiers dans la détermination du niveau de la rémunération.

\section{Critères internes}

Les critères internes, comme l'appellation elle-même le suggère, servent surtout à situer chacun des membres du groupe dans la structure salariale. Parmi ces critères, on retrouve d'abord et avant tout l'expérience et le niveau des qualifications. L'auteur n'est pas clair sur le sujet, mais il semble bien que toute la structure salariale s'échafaude à peu près partout autour de ces deux critères: différents niveaux de qualifications définissent différentes échelles de traitements, les échelons sur chaque échelle étant fonction de l'expérience. C'est du moins le type de structure qui existe au Québec chez les enseignants, comme d'ailleurs chez d'autres catégories de personnel qui bénéficient, par exemple, d'un plan de carrière.

Une question cruciale vient ici à l'esprit: Comment l'enseignant est-il classifié? Ou en d'autres termes, comment est-il intégré dans la structure salariale? On sait qu'au Québec l'enseignant du niveau primaire ou secondaire est classifié en fonction unique et directe du nombre de ses années de scolarité. Mais, l'auteur rapporte que «la méthode que l'on rencontre le plus fréquemment comporte le rattachement du niveau des qualifications requises, au niveau de l'enseignement dispensé». (p. 19) Le lecteur aura sans 
doute compris qu'une telle pratique s'éloigne de façon marquée de celle qui prévaut au Québec où l'enseignant est classifié en fonction de sa scolarité et non du niveau de l'enseignement qu'il dispense. ${ }^{2}$ Dans ce dernier cas, le niveau de l'enseignement exige un certain niveau de qualifications et pas plus, en ce sens que l'enseignant ne sera pas payé davantage, même s'il possède un niveau de qualifications supérieur à celui exigé par le niveau d'enseignement qu'il occupe. L'auteur ajoute cependant qu'on relève des signes d'un intérêt grandissant pour une méthode plus directe pour relier les traitements aux qualifications (comme au Québec).

Avant de passer à l'autre critère interne principal, il convient de mentionner que la matière enseignée joue parfois un certain rôle dans l'intégration des enseignants dans les échelles de traitement ou que, sans influencer l'intégration, elle peut donner lieu à une prime. Des considérations analogues peuvent être faites en relation avec les responsabilités.

En ce qui concerne l'expérience, «tous les systèmes scolaires ou presque tous reconnaissent par le placement à un échelon plus élevé la valeur, chez un enseignant, de son expérience dans la profession.» (p. 23) Il s'agit surtout de l'expérience acquise dans l'enseignement, mais on tient compte aussi, à des degrés divers, de l'expérience acquise en dehors de la profession. On pourrait s'interroger ici sur la relation entre le nombre d'années de service dans l'enseignement et la compétence.

De plus, un petit nombre de pays retiennent le critère âge (présumément comme approximation de l'expérience). Enfin, les états de services, au sens de mérite, sont pris en compte dans un certain nombre de systèmes.

Sans sous-estimer l'importance des critères internes, il faut quand même voir que le niveau réel des traitements dépend surtout des critères externes; comme on l'a écrit plus haut, les critères externes affectent principalement la position d'un enseignant relativement aux autres de son groupe.

\section{Critères externes}

Tout en ayant raison d'écrire que les facteurs externes ne se prêtent pas si aisément que les facteurs internes à une évaluation objective, l'auteur nous surprend au premier abord en n'identifiant ici que deux critères externes: le critère de comparabilité et les critères découlant de la politique gouvernementale. Il faut dire cependant que l'auteur discute ailleurs, au chapitre 5 , de deux autres critères du genre, à savoir le coût de la vie et le niveau général des salaires. Il apparaît en particulier surprenant, surtout à

2 L'auteur n'indique pas de façon explicite comment sont mesurées les qualifications. D'une part, son texte qui traite du niveau des qualifications semble clairement laisser entendre (voir pp. 18-22) que celui-ci se mesure généralement par le nombre d'années d'étude. D'autre part, il écrit que «les systèmes dans lesquels les échelles de salaires reflètent le nombre d'années d'études ou de formation constituent à cet égard des exceptions notoires». (p. 60) Il s'agit là d'une contradiction, au moins en apparence. 
partir de l'expérience québécoise des dernières négociations, que l'auteur ne mentionne pas de façon spécifique l'influence immense que peut avoir la politique salariale d'un gouvernement vis-à-vis ses propres employés, dans la détermination des conditions de travail des enseignants. Nous reviendrons ci-dessous sur l'omission du pouvoir de négociation.

Examinant d'abord le critère de comparabilité, il convient de souligner que, l'allocation des ressources se faisant par le mécanisme des prix dans une économie de marché, cela va de soi que les enseignants, comme tout autre travailleur, compareront les conditions de travail offertes par la profession enseignante avec celles offertes par d'autres professions. Par ailleurs, les employeurs, quels qu'ils soient, offriront des conditions suffisantes pour obtenir les travailleurs requis, mais non des conditions inutilement élevées. Il existe des signaux qui indiquent aux employeurs que leurs conditions sont insuffisantes, pour ne mentionner que les difficultés de recrutement et les taux de départs (et possiblement de roulement) élevés.

Bien sûr, parmi les conditions de travail, le taux de traitement de base occupe une place privilégiée dans les comparaisons en question. Cependant, il faut comparer entre elles des "choses comparables», en l'occurence des travailleurs dont les caractéristiques pertinentes sont suffisamment semblables. La difficulté pour les enseignants, c'est de trouver des travailleurs comparables. Pour contourner cette difficulté, les comparaisons sont souvent effectuées sur la base des qualifications mesurées, par exemple, par les diplômes. Le Québec ne semble pas se distinguer des autres pays à cet égard. Il convient peut-être d'ajouter que les comparaisons possèdent des vertus centripètes et qu'elles tendent donc au nivellement des salaires.

Relativement aux critères découlant de la politique gouvernementale, l'auteur mentionne ici certains facteurs que des gouvernements peuvent considérer dans leur politique salariale des enseignants. Par exemple, un gouvernement peut désirer améliorer la position relative de ses enseignants pour différentes raisons. La capacité de payer d'un gouvernement peut aussi devenir critique, à la suite, entre autres facteurs, d'une augmentation des coûts de l'éducation et en particulier des augmentations de salaire.

On observe enfin le silence de M. Bell sur un facteur qui revêt toujours une signification cruciale dans toute négociation, soit le pouvoir de négociation. Ceci est d'autant plus vrai que ce facteur colorie en un sens tout le processus de négociation. A la défense de l'auteur, il faut cependant souligner que le pouvoir de négociation n'est pas un critère de détermination de la rémunération au même sens que les comparaisons de salaires: ceci tient essentiellement à la quasi impossibilité de mesurer le pouvoir de négociation.

Comme on l'a déjà signalé ci-dessus, la première partie que nous venons de recenser constituait une sorte de longue introduction au sujet central de l'ouvrage du B.I.T., soit ce en quoi consiste la rémunération des enseignants. 
Le chapitre 3 se rapporte à la structure des traitements du personnel enseignant, le chapitre suivant aux suppléments de traitement et le chapitre 5 à l'adaptation des traitements du personnel enseignant aux mouvements du coût de la vie ou du niveau général des salaires. Le dernier chapitre fait un essai de comparaison internationale relativement aux gains des enseignants.

CHAPITRE 3: LA STRUCTURE DES TRAITEMENTS DU PERSONNEL ENSEIGNANT.

L'auteur signale d'abord que «l'un des traits les plus remarquables de la rémunération des enseignants est la prédominance du système des échelles de traitements». (p. 37) En d'autres mots, les enseignants semblent généralement bénéficier d'une sorte de plan de carrière, avec tous les avantages d'un tel plan dans la profession enseignante.

La classification des enseignants a suffisamment été couverte plus haut pour ne pas avoir à y revenir ici. Ce qu'il importe de connaître maintenant, ce sont les cheminements que peuvent suivre les enseignants, une fois qu'ils ont été intégrés dans la structure salariale. A cet égard, malgré la très grande qualité de l'ouvrage en général, il convient de regretter que l'auteur n'ait pas fait ressortir assez clairement la distinction capitale entre les augmentations générales et les augmentations individuelles de traitement. On sait qu'un employé profite d'abord de l'augmentation générale des salaires, puis généralement d'une augmentation individuelle due, entre autres, à l'accès à un échelon supérieur (ou augmentation statutaire) ou à une échelle supérieure (ou reclassement).

En égard aux augmentations individuelles, il importe de savoir, en premier lieu, si les enseignants ont accès à une échelle supérieure de traitement, et si oui, à quelles conditons et, en deuxième lieu, ce qui permet aux enseignants de gravir les échelons de leur échelle actuelle. Il n'est pas indifférent non plus de connaître les différences inter-échelons et interéchelles.

Pour ne pas trop allonger son texte, l'auteur n'a dégagé que deux catégories-types d'enseignants, une catégorie comprennant les moins bien rémunérés et une autre les mieux rémunérés. ${ }^{3}$ Tout en étant sans doute fondée, une telle décision fait perdre une information très intéressante, à savoir le nombre d'échelles existant dans les structures de traitements. Par exemple au Québec, il existe huit échelles (en 1977-1978), la première correspondant à moins de 14 années de scolarité, la deuxième à 14 années et ainsi de suite jusqu'à la huitième échelle qui correspond à 20 années de scolarité et plus. Avec des différences inter-échelles d'environ 9,0\%, cet aspect de la structure salariale du Québec avantage nettement les enseignants les plus fortement scolarisés. Or, dans l'ouvrage du B.I.T., non

3 D'une façon plus précise, il s'agit, d'une part, des enseignants ayant deux années d'études post-secondaires et, d'autre part, des enseignants porteurs d'un diplôme universitaire et comptant une ou deux années de formation spécialisée. (Voir p. 44). 
seulement ne connaît-on pas le nombre d'échelles dans les structures salariales des pays couverts, mais le peu d'information sur les différences entre les échelles des moins bien et des mieux rémunérés semble difficile à interpréter. Encore à la défense de l'auteur, il faut quand même souligner le fait que la promotion à un échelon supérieur est de loin plus fréquente et probable que la promotion à une échelle supérieure.

Dès lors, l'avantage ci-haut mentionné pour les plus scolarisés du Québec vaut surtout lors de leur intégration dans les échelles, ceci ne signifiant par pour autant une sous-estimation de l'avantage en cause. Un certain ordre de grandeur est heureusement disponible quant à l'écart entre les moins bien et les mieux rémunérés. D'après l'étude, l'écart entre les minima des deux échelles (extrêmes, semble-t-il) est rarement inférieur à $5 \%$; dans plusieurs pays (le plus grand nombre, semble-t-il), l'écart varie de 12 à $35 \%$; dans d'autres, les pourcentages correspondants sont 40 et 50. Pour les divers systèmes scolaires du Canada, l'auteur parle d'une fourchette de 30 à $50 \%$. Sans en savoir plus long sur la méthode de calcul utilisée, il apparaît hasardeux d'estimer une telle fourchette pour le Québec. Seul l'auteur pourrait nous renseigner avec confiance sur le sujet. On a déjà vu toutefois que l'écart entre les minima de deux échelles consécutives au Québec se situe autour de $9 \%$ et de plus qu'il existe huit échelles (jusqu'en 1977-1978 inclusivement). L'écart entre les minima des première et huitième échelles atteint donc près de $80 \%$. Mais, cet écart n'est peut-être pas exprimé en termes comparables avec ceux de l'étude de M. Bell.

Attardons-nous maintenant avec l'auteur aux caractéristiques des échelles prises individuellement. En rapport avec les périodes couvertes par les échelles, l'auteur a pu dégager deux groupes principaux de pays. Dans un premier, comprenant le Canada, la période varie de 8 à 15 ans. Dans l'autre groupe, plus important en nombre, les échelles s'étendent sur 20 ans et plus. Or, on sait que les échelles des enseignants du Québec ont une durée de 15 ans.

Quant à la périodicité des augmentations, dans la plupart des pays qui font l'objet de la présente étude, elle est d'une fois l'an. "Cependant, les augmentations sont attribuées tous les deux ans dans un nombre élevé de pays... Dans certains systèmes d'enseignement, les intervalles sont plus longs encore...» (p. 46) Il est intéressant de noter que la périodicité en question est variable dans un certain nombre de pays, les intervalles ayant une nette tendance à être courts au départ, pour s'allonger à mesure que la carrière progresse. Notons enfin qu'une telle variabilité n'existe pas au Québec, la progression salariale n'étant pas accélérée en début de carrière.

L'importance des augmentations (aussi appelées différences interéchelons) constitue une autre caractéristique majeure des échelles de traitements. Il faut d'abord savoir qu'il existe divers modes de calcul de telles augmentations. Pour fins d'exposition, on peut identifier deux modes principaux: d'après le premier, le montant est uniforme pour tous les échelons, alors que d'après le deuxième mode, c'est le pourcentage qui est uniforme. Il existe bien sûr de nombreuses variantes entre ces deux modes extrêmes. D'après l'auteur, la variété de tels modes de calcul rend toute généralisation difficile. Il ajoute cependant que, dans la majorité des 
systèmes de rémunération qui font l'objet de l'étude, «la progression annuelle moyenne des traitements déterminée par la grille des rémunérations, oscille entre 2,5 et 5 pour cent du traitement à l'échelon le plus bas de l'échelle ou du niveau plancher intermédiaire». (p. 52) Or, les enseignants du Québec connaissent présentement un taux d'augmentation uniforme de $3,45 \%$ pour tous les échelons à l'intérieur de toutes les échelles. ${ }^{4}$ En termes du traitement minimum des échelles, qui sert généralement de point de référence à l'auteur, ce taux uniforme de $3,45 \%$ équivaut à un taux qui progresse tout le long de chaque échelle pour atteindre environ 5,5\% au haut de l'échelle. ${ }^{5}$

Pour terminer l'examen du présent chapitre, un dernier point doit être considéré, soit l'automatisme des augmentations. L'auteur distingue en principe deux manières extrêmes d'attribuer les augmentations. "La première est dictée par une attitude plutôt négative, qui conduit à n'octroyer l'augmentation que si la qualité du travail est prouvée». (p. 57) La seconde manière repose sur l'idée que l'augmentation doit être attribuée automatiquement. En résumé, «la plupart des systèmes scolaires...étudiés appliquent des règles qui, par leur teneur, se placent quelque part entre les deux extrêmes». (p. 57) On sait qu'au Québec les augmentations sont attribuées de façon automatique. Enfin, un peu dans la même veine, il faut s'assurer, dans toute comparaison sur une composante quelconque, que toutes choses sont égales par ailleurs. Par exemple, quand on compare entre eux divers taux d'augmentation de traitement, il faut s'assurer que les périodicités, entre autres caractéristiques, sont les mêmes, car une augmentation de $6 \%$ pour deux ans équivaut à une augmentation d'environ $3 \%$ pour un an.

Il convient sans doute de mentionner que, si les augmentations ne doivent pas être attribuées de façon automatique, des critères de promotion (salariale) doivent être mis de l'avant. On pense naturellement ici aux états de service (mérite), avec toutes ses difficultés de mesure bien connues et, partant, avec toutes les situations inéquitables qui peuvent s'ensuivre. Les états de service ont d'ailleurs déjà été examinés avec les critères internes.

\section{CHAPITRE 4: LES SUPPLÉMENTS DE TRAITEMENT.}

Il va sans dire que les suppléments présentent un intérêt beaucoup moins grand que les caractéristiques des structures salariales. D'une façon générale, les suppléments représentent d'ailleurs une portion assez faible des traitements de base (déterminés par les structures). De plus, les suppléments peuvent épouser différentes formes, ce qui entraîne en soi des difficultés de comparaison. Nous nous contenterons donc généralement de mentionner les différentes formes de supplément. A l'occasion seulement, nous tenterons la comparaison avec le Québec.

4 Des ajustements mécaniques, dus à l'application de la clause d'indexation au coût de la vie, peuvent parfois produire un léger écart.

5 En effet, pour une échelle qui va de $\$ 15010$ à $24132,5,5 \%$ de $\$ 15010$ donne $\$ 825,55$ et $3,45 \%$ de $\$ 24132$ donne $\$ 832,55$. 
Un supplément est parfois accordé pour responsabilités d'ordre administratif, par exemple pour seconder le directeur de son école. En ce qui concerne la rémunération des directeurs, l'auteur écrit qu'il renonce à l'examiner dans le détail, face à la diversité des situations. (p. 62) Au Québec, un tel supplément peut exister dont le montant peut varier d'environ $\$ 500.00$ à environ $\$ 1,100.00$ par année. (Entente entre F.C.S.C.Q. et C.E.Q., 1975-1979, pp. 71-72)

Un supplément peut aussi être versé pour des responsabilités liées au type de l'établissement. L'auteur réfère par exemple à des écoles dont les élèves souffrent de handicaps divers. Nous comprenons qu'au Québec un nombre plus petit d'élèves par classe tient lieu de supplément, en un sens.

Des indemnités dites professionnelles sont allouées dans quelques pays, mais apparemment pas au Québec. Il peut aussi exister des indemnités pour activités additionnelles, telle la surveillance des élèves durant les récréations. Au Québec, de telles activités semblent prévues dans la tâche "normale» de l'enseignant. L'auteur ne semble pas citer de systèmes scolaires qui verseraient un supplément aux enseignants qui enseigneraient à des classes plus nombreuses. Au Québec, celles-ci sont prises en compte dans la distribution des tâches connexes à l'enseignement.

Les heures supplémentaires peuvent donner lieu à un supplément de traitement, dans bon nombre de pays. Dans un certain nombre d'autres par contre, on ne prévoit pas de rémunération des heures supplémentaires; on semble plutôt avoir recours à du personnel de remplacement. Une telle pratique existe au Québec, mais les conventions collectives de 1975-1979 contiennent une clause (8-3.04) prévoyant la rémunération du surtemps. De plus, l'auteur mentionne de façon distincte les activités ne relevant pas de l'enseignement proprement dit. En gros, elles semblent pouvoir être considérées comme les activités additionnelles déjà vues. La même chose semble également vraie pour des éléments autres que l'activité professionnelle.

Enfin, les deux types suivants de supplément offrent un intérêt de beaucoup supérieur aux types précédents. Il s'agit d'abord des suppléments en rapport avec le lieu de résidence, et plus particulièrement avec l'isolement. (Par ailleurs, "dans certains systèmes scolaires, l'accomplissement d'une période de service dans une région rurale est une condition préalable de l'avancement au-delà d'un certain seuil».) (p. 73) D'après l'étude, le montant de tels suppléments "peut varier dans des proportions considérables». (p. 74) Au Québec, il existe quatre (4) zones d'éloignement dans lesquelles les enseignants reçoivent un supplément qui peut varier de \$1 291 à $\$ 1935$. De plus, des primes de rétention peuvent être versées aux enseignants de certaines autres. L'étude nous apprend que des taux supérieurs à ceux du Québec, par exemple, sont appliqués dans certains pays. (Il existerait aussi certains systèmes scolaires qui accordent des suppléments pour la résidence dans des centres urbains, le coût de la vie étant généralement plus élevé dans les grands centres urbains.)

Enfin, il existe bien sûr des prestations supplémentaires de caractère social, plus souvent appelées avantages sociaux dans nos milieux. Il s'agit, par exemple, de contributions des employeurs à des régimes d'assurance 
contre des risques tels que la retraite, le décès, la maladie, etc. L'auteur écrit qu'une telle pratique est très répandue aux États-Unis et au Canada. Malheureusement, aucune quantification n'est faite par l'auteur de la valeur de tels avantages ou suppléments par rapport à la rémunération de base.

\section{CHAPITRE 5: ADAPTATION DES TRAITEMENTS AUX MOUVEMENTS DU COÛT DE LA VIE OU DU NIVEAU GÉNÉRAL DES SALAIRES}

Il convient peut-être de rappeler ici que les chapitres 3 et 4 se rapportaient aux augmentations individuelles de traitement, alors que le présent chapitre est relié aux augmentations générales.

L'auteur a certainement raison de parler de l'adaptation des traitements aux mouvements $d u$ coût de la vie. A ce sujet, il écrit que «les autorités... reconnaissent, en règle générale, que les enseignants ont droit à une compensation de l'augmentation du coût de la vie». (p. 82) Malgré cela, certains pays ne disposent d'aucun mécanisme d'adaptation du genre.

D'autres possèdent soit une procédure ad hoc, soit un mécanisme préétabli pour assurer une révision régulière des traitements en fonction du coût de la vie. Encore ici, il est malheureux qu'on ne trouve pas, dans l'étude, une certaine quantification de la protection que possèdent les enseignants contre l'augmentation du coût de la vie. Il n'est peut-être pas suffisant pour le lecteur d'apprendre que «les systèmes... permettent de limiter le plus possible l'érosion du pouvoir d'achat...» (p. 86) Les enseignants du Québec, eux, possèdent une excellente protection du genre, depuis au moins 1971-1972. Effectivement, les traitements ont été indexés en 1975-1976 pour tenir compte de l'augmentation de l'indice des prix à la consommation depuis 1971-1972 et ils sont aussi indexés chaque année depuis 1975-1976. ${ }^{6}$ Sans entrer dans le détail ici, puisque le présent texte vise à comparer le Québec avec d'autres pays, il convient de souligner que les enseignants du Québec bénéficient présentement d'une excellente protection contre l'inflation.

Le dernier point que l'auteur a couvert dans son étude se rapporte à l'ajustement au niveau général des salaires. La couverture du sujet semble des plus sommaires. On lit, par exemple, qu' 'on s'efforce, dans quelques pays, d'aligner les traitements des enseignants sur le niveau général des salaires» (p. 86), mais que ces pays sont en très petit nombre. Il apparaît enfin fort surprenant de lire qu' «aucun pays ne paraît avoir aligné formellement les traitements du personnel enseignant sur le mouvement général de la productivité». (p. 88) En effet, les enseignants du Québec, entre autres, ont vu leurs traitements alignés sur le mouvement général de la productivité, au moins en très bonne partie (sans plus de détails, pour ne pas entrer ici dans des considérations techniques, pour ne pas dire subjectives, sur la mesure de la productivité).

6 De 1971-1972 à 1974-1975, des montants forfaitaires étaient versés, lesquels faisaient plus que compenser pour l'augmentation des prix à la consommation. 
Il convient enfin de noter qu'on ne peut pas logiquement s'attendre à trouver des systèmes scolaires qui adapteraient les traitements de leurs enseignants aux mouvements à la fois des prix à la consommation et du niveau général en dollars courants, puisque le second tient déjà compte, au moins partiellement, du premier.

\section{CHAPITRE 6: QUELS SONT LES GAINS DES ENSEIGNANTS? UN ESSAI DE COMPARAISON INTERNATIONALE.}

Dans un premier temps, l'auteur fait des considérations fort intéressantes sur différentes mesures possibles pour fins de comparaison internationale. Sans nous attarder sur le sujet, nous donnons raison à $\mathrm{M}$. Bell d'avoir choisi les gains des enseignants relativement à ceux des travailleurs des industries manufacturières. L'auteur a donc évité, entre autres choses, de comparer directement les revenus de même que le pouvoir d'achat de ceux-ci. L'auteur insiste aussi sur le caractère indicatif des comparaisons, d'autant plus que celles-ci ne portent que sur les deux catégories d'enseignements déjà dégagées, soit les moins et les plus scolarisés. De plus, des mesures comparables n'ont pu être calculées pour plusieurs pays, faute de données suffisantes, pour ne mentionner que les États-Unis, l'U.R.S.S., la Belgique, le Danemark, l'Italie, le Japon, la Nouvelle-Zélande et la République démocratique allemande. L'essai de comparaison internationale tient aussi compte de l'indice des prix à la consommation.

Etant donné que les mesures en question sont fournies et analysées par l'auteur pour chacun des pays couverts, il n'est certes pas pensable de faire état ici de façon détaillée de ce chapitre, des plus intéressants par ailleurs. Nous nous contenterons donc de rendre compte de quelques conclusions dégagées par $\mathrm{M}$. Bell lui-même.

D'abord, dans la majorité des pays analysés, le traitement initial d'un enseignant parmi les moins scolarisés «était sensiblement inférieur au gain moyen dans les industries manufacturières». (p. 124) De plus, dans presque tous les pays, "la rémunération du personnel enseignant a augmenté plus rapidement et parfois même beaucoup plus rapidement que l'indice officiel des prix à la consommation». (p. 124) Enfin, "dans de nombreux pays, les gouvernements ont été amenés à prendre des mesures spéciales en vue de rétablir la condition matérielle du personnel enseignant dans le cadre des hiérarchies nationales de salaires»). (p. 125)

CONCLUSION À L'EXAMEN DE L'OUVRAGE DU B.I.T.

En gros, M. Bell a effectué une très bonne étude de la rémunération des enseignants. D'une façon générale, toute personne intéressée à la question peut compléter par elle-même l'étude du B.I.T., en comparant les données pour son propre système scolaire (comme nous avons tenté de le faire) avec celles disponibles dans l'étude. Naturellement, tout lecteur intéressé déplorera l'absence ou l'insuffisance de données sur quelques points impor- 
tants tels que le nombre des échelles de traitements et les clauses d'indexation des salaires. De plus, une distinction plus nette entre les augmentations individuelles et générales aurait sans doute facilité la lecture du texte. Le lecteur aurait pu également souhaiter un peu plus grand effort de quantification, par exemple, des avantages sociaux. Evidemment, de telles critiques sont faciles à exprimer par le lecteur. Cependant, il faut admettre que, dans une étude comme celle conduite par M. Bell, il n'est pas aussi facile d'en tenir compte.

\section{Employment Problems of the Native People in Ontario}

\section{Harish C. Jain}

This paper describes and analyzes some of the reasons for the native peoples lack of access to economic opportunities and employment relative to the non-native population of Ontario. In order to overcome the labour market problems faced by the native people living on-reserves and offreserves, some policy options are suggested.

\section{DEMOGRAPHIC CHARACTERISTICS OF THE NATIVE PEOPLE IN ONTARIO}

Population: Status Indians accounted for approximately 1 percent (approximately 62,000) of Ontario's population and 1.4 percent $(295,000)$ of Canada's population in $1971 .^{1}$ When non-status Indians are included, native people could well represent almost five percent of both Ontario's and Canada's population. ${ }^{2}$

At present, both the Ontario and the Canadian Indian population is young and growing rapidly. ${ }^{3}$ This trend is expected to continue in the

* JAIN, Harish C., Personnel and Industrial Relations Area, Faculty of Business, McMaster University, Hamilton, Ontario.

** Detailed tables on the demographic characteristics of the Native people in Ontario are provided in the report entitled Labour Market Problems of Native People in Ontario by Harish C. Jain, Faculty of Business, McMaster University, Hamilton, Ontario, Research and Working Paper Series 139, December 1977, pp. 124. These tables are available on request.

11971 Census of Canada.

2 FRIDERES, J.S., Canada's Indians: Contemporary Conflicts, Scarborough; Ontario, Prentice-Hall, 1974, p. 13.

3 Ontario Statistics, 1976, Vol. 1, Toronto, Ontario. 